\title{
Strawberry Plant Extracts Stimulate Secondary Conidiation by Colletotrichum acutatum on Symptomless Leaves
}

\author{
L. F. S. Leandro, M. L. Gleason, F. W. Nutter, Jr., S. N. Wegulo, and P. M. Dixon
}

First, second, third, and fourth authors: Department of Plant Pathology; and fifth author: Department of Statistics, Iowa State University, Ames 50011.

Accepted for publication 28 April 2003.

\begin{abstract}
Leandro, L. F. S., Gleason, M. L., Nutter, F. W., Jr., Wegulo, S. N., and Dixon, P. M. 2003. Strawberry plant extracts stimulate secondary conidiation by Colletotrichum acutatum on symptomless leaves. Phytopathology 93:1285-1291.

Conidial suspensions of Colletotrichum acutatum were prepared in 1:27, 1:45, and 1:81 (wt/vol) dilutions of an extract of strawberry (cv. Tristar) flowers or leaves in water. Strawberry leaves and plastic coverslips were sprayed with the conidial suspensions, incubated at $25^{\circ} \mathrm{C}$ and continuous wetness for $48 \mathrm{~h}$, and the number of conidia and appressoria were counted. In another experiment, leaves and coverslips were sprayed with a conidial suspension in water, incubated for $72 \mathrm{~h}$ to

incubated for $48 \mathrm{~h}$ at $25^{\circ} \mathrm{C}$ and continuous wetness, and the number of conidia and appressoria were counted. Flower extracts significantly $(P \leq$ 0.05 ) increased the number of conidia on leaves and coverslips compared with water, both when conidia were applied with the extracts and when the extracts were applied to $C$. acutatum populations exposed to dryness for up to 2 weeks. Application of flower extracts resulted in up to 10- and 16-fold increases in conidia on leaves and coverslips, respectively. Number of conidia increased more when exposed to flower extracts than to leaf extracts. Production of appressoria was not significantly affected by flower or leaf extracts. Our results suggest that inoculum levels of $C$. acutatum on foliage may increase during flowering of strawberry plants.
\end{abstract} establish C. acutatum populations, and placed in a growth chamber under dry conditions for up to 6 weeks. At each sampling time, leaves and coverslips were sprayed with flower extracts, leaf extracts, or water,
Additional keywords: phyllosphere ecology, secondary conidiation, strawberry anthracnose.
The anthracnose pathogen, Colletotrichum acutatum J. H. Simmonds, is a serious threat to strawberry production worldwide $(6,16,27)$. All strawberry plant parts are susceptible to infection $(8,12,27)$, although fruit rot is particularly damaging in production fields, where yield losses can be severe $(11,16,27,33)$. Anthracnose development in strawberry nurseries not only reduces daughter plant production $(6,28)$ but also has serious consequences for pathogen dissemination on contaminated transplants $(5,6,8,9)$. Once $C$. acutatum is introduced into production fields, disease outbreaks can be very difficult to manage because available tactics are only partially effective $(12,16,27)$. The use of pathogen-free propagation material, therefore, is highly desirable $(16,17,27)$ but is difficult to implement due to the lack of adequate detection and certification schemes for $C$. acutatum on symptomless plants $(16,27)$.

Epiphytic behavior of $C$. acutatum on symptomless foliage may be important in the strawberry anthracnose disease cycle $(7,13$, 23). C. acutatum recently has been shown to produce secondary conidia on strawberry leaves in the absence of symptom development (13), and to persist on symptomless foliage of strawberry $(7,13,34)$ and weeds (7) for several weeks. Symptomless leaves, therefore, may serve as an inoculum source for strawberry anthracnose $(7,13)$. Similarly, symptomless foliage of tomato (20) and citrus $(1,30)$ has been reported to provide inoculum for diseases caused by $C$. coccodes and $C$. acutatum, respectively.

The behavior of Colletotrichum spp. on plant surfaces may be affected by several factors, including the presence of host exudates $(10,22,25)$. On citrus, flower extracts play an important role in post-bloom fruit drop caused by $C$. acutatum $(1,30,35)$. Water-

Corresponding author: M. L. Gleason; E-mail address: mgleason@iastate.edu

Publication no. P-2003-0812-01R

(C) 2003 The American Phytopathological Society soluble flower extracts stimulate appressoria surviving on the leaf surface to germinate and produce secondary conidia without development of typical lesions or fruiting structures. These conidia then serve as initial inoculum for citrus flower infections $(1,30)$. The potential for $C$. acutatum to produce secondary conidia on symptomless strawberry leaves has been characterized under a range of temperatures and intermittent wetness regimes $(13,14)$. However, it is not known if flower extracts can stimulate inoculum production by $C$. acutatum on strawberry foliage $(1,30)$. A better understanding of $C$. acutatum behavior in the presence of plant extracts may shed light on the role of symptomless foliage as a source of inoculum for strawberry anthracnose.

The objective of this study was to determine the effects of strawberry plant extracts on conidial germination, appressorial development, and secondary conidiation of $C$. acutatum on strawberry leaves. A preliminary report has been published (15).

\section{MATERIALS AND METHODS}

Plant production and maintenance. Day neutral strawberry crowns (cv. Tristar) were planted in 15-cm-diameter plastic pots containing a 1:2:1 mixture of peat, perlite, and soil, and grown in a greenhouse maintained at $25 \pm 10^{\circ} \mathrm{C}$ with a 16-h photoperiod. The plants were drip irrigated and fertilized with a solution of a 21-5-20 fertilizer (400 ppm of N; Miracle Gro Excel; The Scotts Company, Marysville, $\mathrm{OH}$ ) as needed. Fully opened strawberry flowers were collected during the week prior to extract preparation and stored in the refrigerator until use. Healthy, mature strawberry leaves were collected and used for extract preparation on the same day. Strawberry plants grown in the same way were used for inoculations in all experiments.

Inoculum production. Cultures of $C$. acutatum isolate HF-ac98, obtained from strawberry fruit in Iowa (13), were started by 
depositing infested silica gel crystals on potato dextrose agar (PDA) plates and culturing at $25^{\circ} \mathrm{C}$ in the dark for 5 to 7 days. Inoculum then was increased by spreading conidia collected from the PDA cultures onto strawberry fruit agar (SFA) (13) and incubating for 7 days at $25^{\circ} \mathrm{C}$ in the dark.

Extract preparation. Concentrated flower extract (1:9, wt/vol) was prepared by grinding $7 \mathrm{~g}$ of petals, pistils, and stamens (sepals and peduncles were discarded) in $63 \mathrm{ml}$ of sterile deionized water (SDW) using a mortar and pestle. The resulting flower pulp was poured through four layers of sterile cheesecloth into a flask, and the filtrate was centrifuged at $10,000 \mathrm{rpm}(12,100 \times g)$ for $5 \mathrm{~min}$. The supernatant was vacuum filtered through Whatman No. 1 filter paper (Maidenstone, UK) to remove larger particles, then filter-sterilized through a 0.45 - $\mu$ m-diameter millipore membrane (Fisherbrand, Pittsburgh, PA). Concentrated leaf extract (1:9, wt/vol) was prepared by macerating $16 \mathrm{~g}$ of leaves, without petioles, in $144 \mathrm{ml}$ of SDW for $1 \mathrm{~min}$ in a blender. The leaf pulp was passed through cheesecloth, centrifuged, and vacuum filtered as described for the flower extract.

Germination of conidia in flower and leaf extracts. Conidial suspensions of $C$. acutatum were prepared by flooding SFA culture plates with $5 \mathrm{ml}$ of SDW, scraping with a rubber policeman to dislodge conidia, and filtering the resulting suspension through two layers of cheesecloth into a flask. The conidial concentration of the suspension then was calculated using a hemacytometer. One part of concentrated $(1: 9, \mathrm{wt} / \mathrm{vol})$ flower or leaf extract was added to two, four, and eight parts (vol/vol) of a mixture of equal volumes of conidial suspension and SDW to make up the desired volume, resulting in final extract dilutions of 1:27, 1:45, and 1:81 (wt/vol), each with $5 \times 10^{5}$ conidia $\mathrm{ml}^{-1}$. Flower and leaf extract dilutions containing conidia will be referred to as $\mathrm{F} 1: 27^{\mathrm{c}}, \mathrm{F} 1: 45^{\mathrm{c}}$, $\mathrm{F} 1: 81^{\mathrm{c}}$ and $\mathrm{L} 1: 27^{\mathrm{c}}, \mathrm{L} 1: 45^{\mathrm{c}} \mathrm{L} 1: 81^{\mathrm{c}}$, respectively. In addition, a $5 \times$ $10^{5}$ conidia $\mathrm{ml}^{-1}$ suspension was prepared in SDW as a control, and will be referred to as Water ${ }^{\mathrm{c}}$.

The experiment consisted of seven treatments (Water ${ }^{\mathrm{c}}, \mathrm{F} 1: 27^{\mathrm{c}}$, $\mathrm{F} 1: 45^{\mathrm{c}}, \mathrm{F} 1: 81^{\mathrm{c}}, \mathrm{L} 1: 27^{\mathrm{c}}, \mathrm{L} 1: 45^{\mathrm{c}}$, and $\left.\mathrm{L} 1: 81^{\mathrm{c}}\right)$ and three sampling times $(0,24$, and $48 \mathrm{~h}$ after inoculation), and followed a randomized complete block design (RCBD) with two replications. Six strawberry plants, each with two 7- to 14-day-old tagged leaves, and six petri dishes, each containing four plastic coverslips on moistened filter paper, were arbitrarily assigned to each treatment, and spray inoculated with the corresponding conidial suspension using an atomizer (DeVilbiss Air Compressor, 571 Series, Somerset, PA). Two plants and two petri dishes per treatment were sampled immediately after inoculation $(0 \mathrm{~h})$ to estimate initial conidial populations applied in each treatment. The remaining petri dishes and plants were incubated in dew chambers at $25^{\circ} \mathrm{C}$ in darkness, according to an RCBD with two blocks. Two plants and two petri dishes per treatment were removed from the chambers at 24 and $48 \mathrm{~h}$ after inoculation.

Leaves and coverslips sampled at each time were allowed to dry before preparation for microscopic observation. Two leaf disks were excised from each of the two tagged leaves per plant using a 1-cm-diameter cork borer. Each leaf disk was mounted on a drop of Hoyer's medium (4) and stained with a drop of aniline blue in lactophenol, and a glass coverslip was placed on top. Inoculated plastic coverslips were placed directly on a drop of aniline blue, with the inoculated side facing downward. A total of eight leaf disks (two disks by two leaves by two plants) and eight coverslips (four coverslips by two petri dishes) were prepared per treatment at each sampling time. The number of ungerminated and germinated conidia and the number of appressoria were counted on 16 microscope fields $(\times 400)$ per leaf disk and coverslip. A conidium was considered germinated when it had formed either a germ tube at least as long as its width, an appressorium, or a conidial phialide. An appressorium was defined as a globose, septum-delimited structure formed either on a hyphal tip or sessile to a conidium. Propagules per unit area (square millimeters) were obtained by dividing the total number of conidia or appressoria counted in each leaf disk or coverslip by the area of 16 microscope fields. The experiment was repeated twice.

Extract effects on established $C$. acutatum populations. Flower and leaf extract dilutions of 1:27 and 1:81 (wt/vol) in water were prepared by adding one part concentrated $(1: 9, \mathrm{wt} / \mathrm{vol})$ extract, prepared as described above, to two and eight parts SDW, respectively. Extract dilutions, without conidia added, will be referred to as F1:27 and F1:81 for the flower dilutions and L1:27 and L1:81 for the leaf dilutions. Extracts were prepared at the beginning of the experiment and kept frozen in vials until needed for application. The experiment consisted of five treatments (Water, F1:27, F1:81, L1:27, and L1:81) and five treatment application times (after $0,1,2,4$, and 6 weeks in a growth chamber), and followed a split-plot design with two blocks, using time as the main-plot effect and treatment as the split-plot effect. In all, 10 strawberry plants, each with two 7- to 14-day-old tagged leaves, and 10 petri dishes, each containing four coverslips on moistened filter paper, were arbitrarily assigned to each time, and two plants and petri dishes were arbitrarily assigned to a treatment within each time. Two additional plants and petri dishes per time were assigned to a pretreatment assessment of $C$. acutatum populations to determine conidial and appressorial populations present at each time before application of water or plant extracts.

A conidial suspension of $C$. acutatum was prepared by flooding SFA culture plates with $5 \mathrm{ml}$ of SDW, scraping with a rubber policeman to dislodge conidia, and filtering the resulting suspension through two layers of cheesecloth into a flask. The suspension was adjusted to $5 \times 10^{5}$ conidia $\mathrm{ml}^{-1}$ with SDW. This suspension was spray inoculated onto leaves and coverslips with an atomizer as described previously. Inoculated material was incubated for $72 \mathrm{~h}$ in dew chambers (100\% relative humidity [RH]) at $25^{\circ} \mathrm{C}$ in darkness, then transferred to a growth chamber at $25^{\circ} \mathrm{C}$ with a 12-h photoperiod after air drying with fans. Leaves and coverslips remained in the growth chamber for 0 to 6 weeks in order to expose $C$. acutatum populations to dryness before application of plant extracts or water. No free water was formed on leaves or coverslips in the growth chamber ( $\mathrm{RH}$ ranged from 35 to $50 \%)$.

After exposure to each dry period $(0,1,2,4$, and 6 weeks), two plants and two petri dishes per treatment were removed from the growth chamber, sprayed with plant extract or water, and immediately placed in a dew chamber at $25^{\circ} \mathrm{C}$ and $100 \% \mathrm{RH}$ for $48 \mathrm{~h}$. Leaves and coverslips were allowed to dry, then prepared for microscopic observation as described above. For each dry period treatment, the two plants and petri dishes assigned to a pretreatment assessment of $C$. acutatum populations were removed from the growth chamber and immediately prepared for microscopic observation without application of water or plant extracts or further incubation. Conidial and appressorial populations were quantified as above and the experiment was repeated once.

Data analysis. The experimental design used for determining the effects of plant extracts on conidial germination was a RCBD with two replications, and the experiment was repeated twice (three runs). However, due to experimental mishaps, only four replications (one from the first two runs of the experiment and two from the third run) yielded data. Based on the lack of experimenttreatment interactions, data from these four replications were combined and analyzed according to an RCBD. Data on effect of extracts on established $C$. acutatum populations were analyzed separately for each run of the experiment because of significant experiment-treatment interactions for some measurements. Data were analyzed separately for each sampling time according to an RCBD with two replications. Data for pretreatment $C$. acutatum populations at each time were not included in this analysis. In both experiments, data were analyzed using the GLM procedure of SAS (SAS Institute, Cary, NC) and treatment effects were 
compared using Fisher's least significant difference test (LSD). Statistical significance was expressed at the $P=0.05$ threshold level.

\section{RESULTS}

Germination of conidia in flower and leaf extracts. Strawberry flower and leaf extracts significantly affected conidial populations of $C$. acutatum when conidia were applied with the extracts to leaves and coverslips. The number of conidia applied at inoculation $(0 \mathrm{~h})$ did not differ significantly among treatments on each substrate (Fig. 1A). After $24 \mathrm{~h}$, there were significantly more conidia on leaves in all flower extract treatments than in water, and conidial numbers were significantly higher in the most concentrated flower extract than in the other dilutions (Fig. 1A). The number of conidia increased 10-fold within $24 \mathrm{~h}$ after inoculation in the most concentrated flower extract, and six- to sevenfold in the more dilute flower extracts (Fig. 1A). Conidial numbers on leaves were significantly higher in the presence of leaf extracts than in water for the two most concentrated dilutions but did not differ significantly among the three dilutions (Fig. 1A). Conidia increased approximately seven- and fourfold in most concentrated and intermediate leaf extract dilutions, respectively, within $24 \mathrm{~h}$ after inoculation (Fig. 1A).

The number of germinated conidia on leaves did not differ among treatments and ranged from 9 to 15 conidia $/ \mathrm{mm}^{2}$. As with total conidia, the number of ungerminated conidia were significantly higher in the flower extract treatments than in water and increased significantly with increasing extract concentration. There were no significant treatment effects on the number of appressoria formed by C. acutatum on leaves (Fig. 1A). Treatment differences observed on leaves $48 \mathrm{~h}$ after inoculation (data not shown) were similar to those at $24 \mathrm{~h}$.

Extract effects on conidial and appressorial numbers on coverslips (Fig. 1B) were similar to those on leaves. At $24 \mathrm{~h}$ after inoculation, conidial numbers on coverslips were significantly higher in flower extracts than in water, and were significantly higher in the most concentrated extract than in the more dilute extracts (Fig. 1B). Conidial numbers increased 16-fold between 0 and $24 \mathrm{~h}$ after inoculation in the most concentrated flower extract, and 9- to 10-fold in the more dilute flower extracts. The two most concentrated leaf extracts significantly increased the total number of conidia compared with water, but there were no differences among leaf extract dilutions (Fig. 1B). The increase in conidial numbers caused by leaf extracts was not as pronounced as with flower extracts. The number of appressoria formed on coverslips did not differ among treatments at either sampling time (Fig. 1B). Treatment differences observed on coverslips $48 \mathrm{~h}$ after inoculation (data not shown) were similar to those at $24 \mathrm{~h}$.

Extract effects on established $C$. acutatum populations. Application of flower extracts to $C$. acutatum populations exposed to different durations of dry conditions significantly affected the total conidial numbers on leaves and coverslips (Fig. 2). There were significantly more conidia on leaves treated with the most concentrated flower extract than in any other treatment after exposure to 0 and 2 weeks of dryness in run 1 of the experiment (Fig. 2A) and after exposure to 1 week of dryness in run 2 (Fig. 2B). Leaf extracts did not affect the number of conidia on leaves compared with the water control (Fig. 2A toB), except after exposure to 2 weeks of dryness in run 1 (Fig. 2A). On coverslips, both flower extract dilutions significantly increased conidial numbers compared with the water control after exposures of 0,1 , or 2 weeks of dryness in run 1 (Fig. 2C) and 0 and 2 weeks of dryness in run 2 (Fig. 2D). Flower extract dilutions differed significantly in their capacity to stimulate secondary conidiation on coverslips after 0 weeks of dryness in both runs of the experiment (Fig. 2C and D). Application of the most concentrated leaf extract to coverslips significantly increased the number of conidia compared with the water control after 0,1 , and 2 weeks of dryness in run 1 (Fig. 2C) and 0 and 2 weeks in run 2 (Fig. 2D), but this effect was less pronounced than with flower extracts. Flower extract effects on conidial numbers became less pronounced with increasing dryness exposure, particularly on leaves where conidial populations tended to decrease over time in all treatments (Fig. 2).

The number of appressoria formed on both leaves and coverslips did not differ significantly among treatments at each time (Fig. 3), except in run 2 on coverslips where water application after 0 weeks of dryness resulted in more appressoria than application of leaf extracts (Fig. 3D). In general, the number of appressoria did not tend to increase or decrease over time (Fig. 3).

\section{DISCUSSION}

This study has shown that inoculum production by $C$. acutatum on symptomless leaves can be strongly stimulated by strawberry
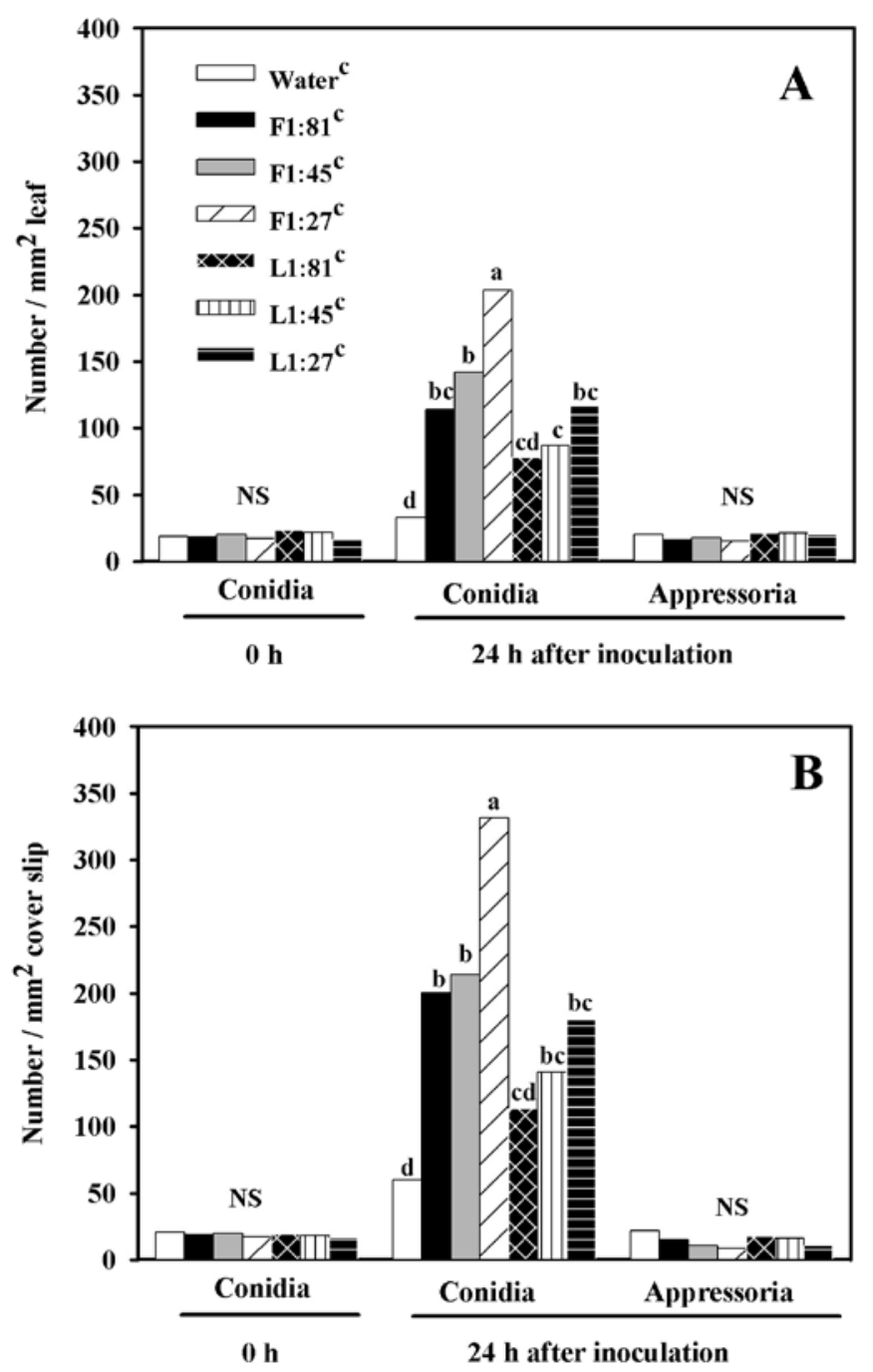

Fig. 1. Colletotrichum acutatum conidia and appressoria on A, strawberry leaves and $\mathbf{B}$, coverslips inoculated with $5 \times 10^{5}$ conidia/ml suspensions in water $\left(\mathrm{Water}^{\mathrm{c}}\right)$, flower extracts $\left(\mathrm{F} 1: 27^{\mathrm{c}}, \mathrm{F} 1: 45^{\mathrm{c}}\right.$, and $\left.\mathrm{F} 1: 81^{\mathrm{c}}\right)$, or leaf extracts $\left(\mathrm{L} 1: 27^{\mathrm{c}}, \mathrm{L1}: 45^{\mathrm{c}}\right.$, and $\left.\mathrm{L} 1: 81^{\mathrm{c}}\right)$ immediately after inoculation $(0 \mathrm{~h})$ or after $24 \mathrm{~h}$ of incubation under continuous wetness at $25^{\circ} \mathrm{C}(24 \mathrm{~h}$ after inoculation). Flower and leaf extracts dilutions are represented by the ratio (wt/vol) of plant material to water. Data presented are means for the four replications included in the analysis. Bars containing the same letter are not significantly different at $P=0.05$ according to Fisher's least significant difference (LSD) test $(n=4)$. NS $=$ no significant differences within the group. LSD values for treatment comparisons within each group are $\mathbf{A}, 9.39,50.21$, and 12.92 for conidia $0 \mathrm{~h}$, conidia $24 \mathrm{~h}$, and appressoria $24 \mathrm{~h}$, respectively, on leaves and $\mathbf{B}$, 5.76, 74.3, and 9.99 for conidia $0 \mathrm{~h}$, conidia $24 \mathrm{~h}$, and appressoria 24h, respectively, on coverslips. 
flower extracts. Secondary conidiation was stimulated by flower extracts both when conidia were germinated in the extracts and when $C$. acutatum populations exposed to dryness for up to 2 weeks were treated with extracts. A similar stimulation occurs on citrus, where flower extracts induce $C$. acutatum appressoria to germinate and produce conidia on leaf surfaces $(1,30,35)$. In our study, secondary conidia remained ungerminated on leaf or coverslip surfaces as indicated by the lack of significant extract effects on the number of germinated conidia, and the small proportion of conidia that were germinated. The fact that flower extracts stimulated secondary conidiation on both leaves and coverslips indicates that this stimulation does not depend on leaf surface characteristics, and that host infection is not required.

C. acutatum conidia typically germinate by forming germ tubes and appressoria $(13,22)$. However, conidia also can germinate by forming conidial phialides, specialized conidiogenous structures that produce secondary conidia without extensive mycelial growth $(13,22,29)$. We recently reported that $C$. acutatum can produce secondary conidia on symptomless strawberry leaves under a range of temperatures and intermittent wetness periods, resulting in up to threefold increases in conidial populations on the leaf surface $(13,14)$. In this study, conidial populations increased by up to 10 -fold on strawberry leaves when germination occurred in the presence of strawberry flower extracts. This suggests that ungerminated conidia exposed to flower extracts have a greater capacity to produce secondary inoculum on strawberry leaves than conidia germinating in water. The cause of the observed stimulatory effect is not clear but may be associated with nutrients or other stimulatory substances in the extracts $(21,22)$. The stimulatory effects of flower extracts on $C$. acutatum multiplication were even more pronounced on coverslips, where conidial populations increased by up to 16-fold. Excess nutrients and microorganisms on the leaf surface may have attenuated the effects of the extracts on leaves compared with coverslips $(21,22)$. Nutrient deficiency, for example, has been associated with induction of secondary conidiation by fungi such as Helminthosporium sativum (3) and $C$. gloeosporoides (26) in response to inhibition of hyphal development $(26,29)$. A stronger effect of nutrients in vitro than on host
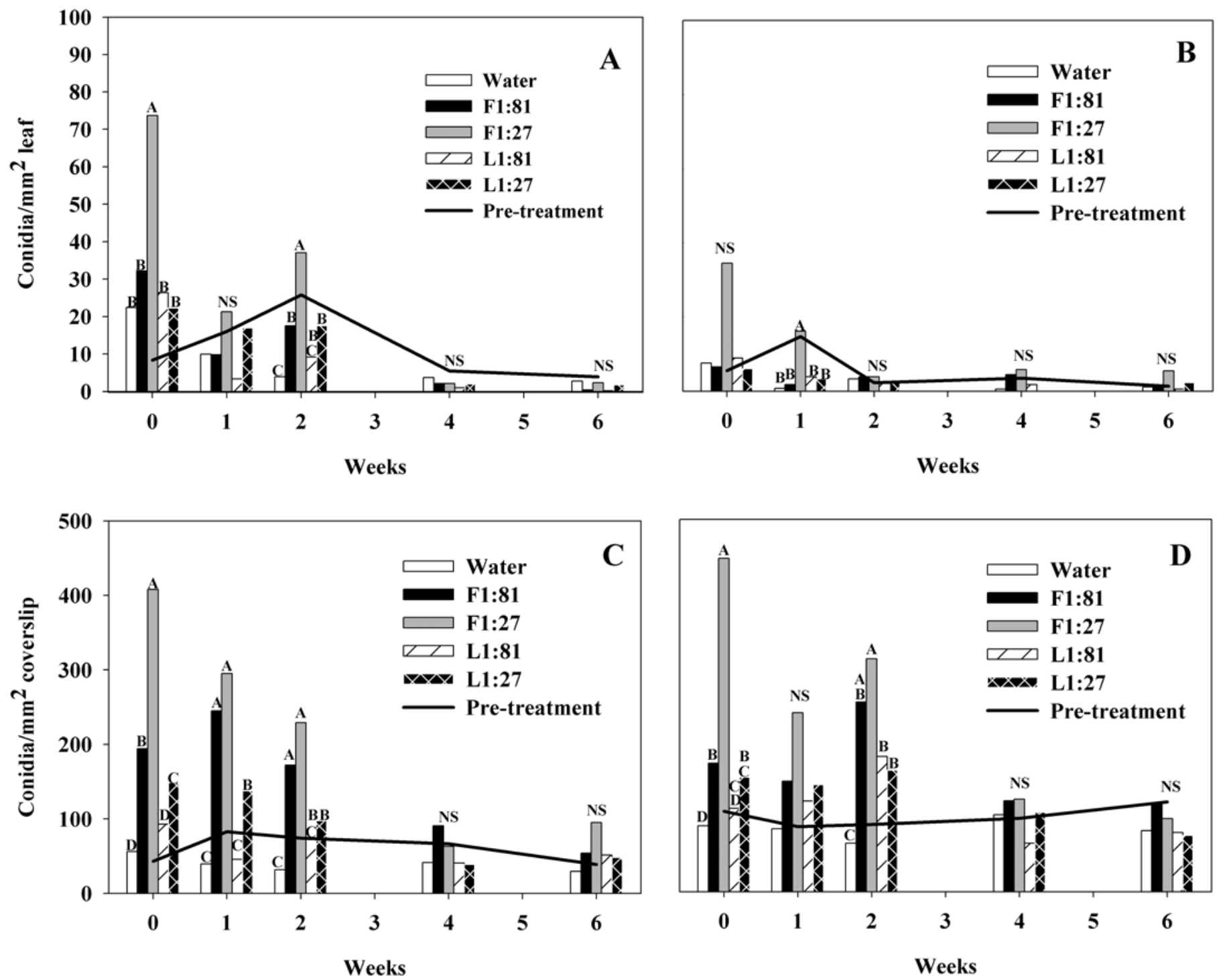

Fig. 2. Colletotrichum acutatum conidia on leaves in $\mathbf{A}$, run 1 and $\mathbf{B}$, run 2 of the experiment, and on coverslips in $\mathbf{C}$, run 1 and $\mathbf{D}$, run 2 . Leaves and coverslips were sprayed with a conidial suspension, incubated for $72 \mathrm{~h}$ at $25^{\circ} \mathrm{C}$ under continuous wetness, and sprayed with water, flower extracts (F1:27 and F1:81), or leaf extracts (L1:27 and L1:81) after 0 to 6 weeks exposure to dryness at $25^{\circ} \mathrm{C}$. After treatment application, leaves and coverslips were incubated for $48 \mathrm{~h}$ under continuous wetness at $25^{\circ} \mathrm{C}$. Flower and leaf extract dilutions are represented by the ratio (wt/vol) of plant material to water. The continuous line represents conidial populations prior to treatment application and incubation. Bars containing the same letter at each time are not significantly different at $P=0.05$ according to Fisher's least significant difference (LSD) test $(n=2)$. NS $=$ no significant differences within the group. Data presented are means for each experiment. LSD values to compare treatment means within sampling times $0,1,2$, 4, and 6 weeks are, respectively A, 25.32, 11.79, 8.79, 3.04, and 4.17; B, 26.84, 9.32, 8.72, 5.69, and 6.37; C, 39.03, 82.88, 65.37, 84.19, and 33.28; and D, 48.92, 121.42, 93.6, 71.02, and 76.73. 
surfaces also was noted by Parbery and Blakeman (22) in germination studies with $C$. acutatum.

Appressorial production by fungi is affected by several factors, including the presence of host exudates $(10,22)$. Production of appressoria by $C$. piperatum, for example, was induced by extracts from red pepper fruit but not from leaves, stems, or green fruit, and was shown to depend on the quality and quantity of nutrients present in the extracts (10). In our study, however, appressorial production by $C$. acutatum occurred on both leaves and coverslips, and was affected significantly only once by strawberry flower or leaf extracts. These results suggest that the substances present in these extracts do not affect appressorial production by $C$. acutatum. The fact that appressoria were formed on coverslips indicates that presence of the host surface is not required for the development of these structures. This result is consistent with previous observations that appressorial production by Colletotrichum spp. is not dependant on specific physical or chemical characteristics of the host $(21,22)$. Zulfiqar et al. (35) reported that $C$. acutatum appressoria surviving on citrus leaves can be induced to germinate and produce secondary conidia by flower extracts, but appressorial germination was not apparent in our study. In previous work, we reported the production of secondary conidia by conidial and hyphal phialides produced shortly after germination $(13,14)$. In this study, however, it was not clear if secondary conidia were produced by phialides that originated from germination of either conidia or resting appressoria.

The ability of flower extracts to stimulate $C$. acutatum conidiation decreased with increasing duration of dry periods, possibly due to increased fungal mortality over time. In contrast, appressoria tended to persist on leaves and coverslips both before and after application of treatments, consistent with their role as survival structures $(18,21)$. The fact that $C$. acutatum could be stimulated to multiply on coverslips and leaves even after 2 weeks of exposure to dry conditions is a clear indication of the durability of C. acutatum propagules and demonstrates that survival does not depend on the presence of the host. C. acutatum survival on inert

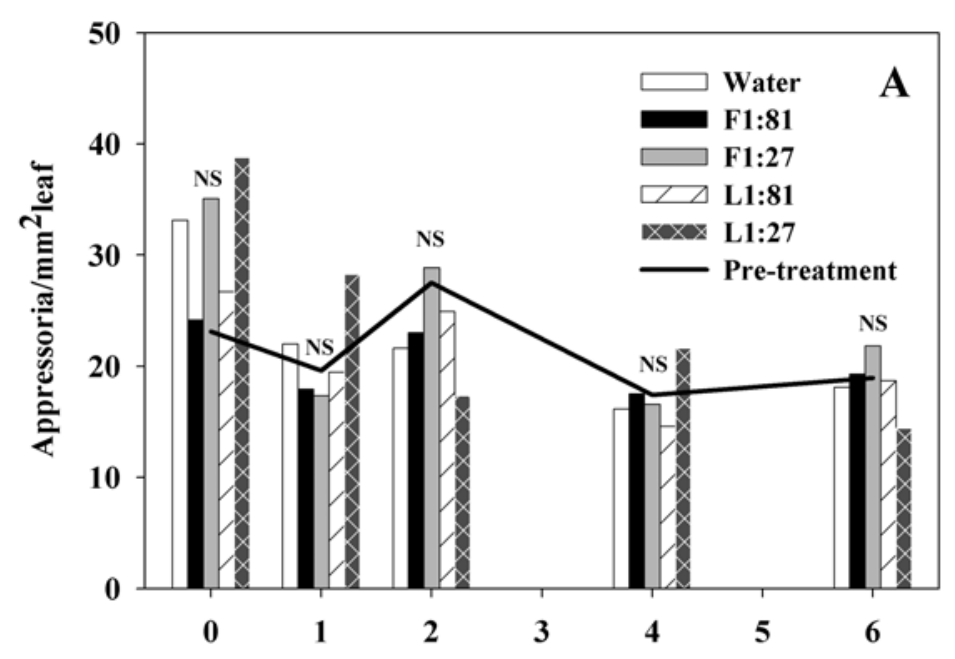

Weeks

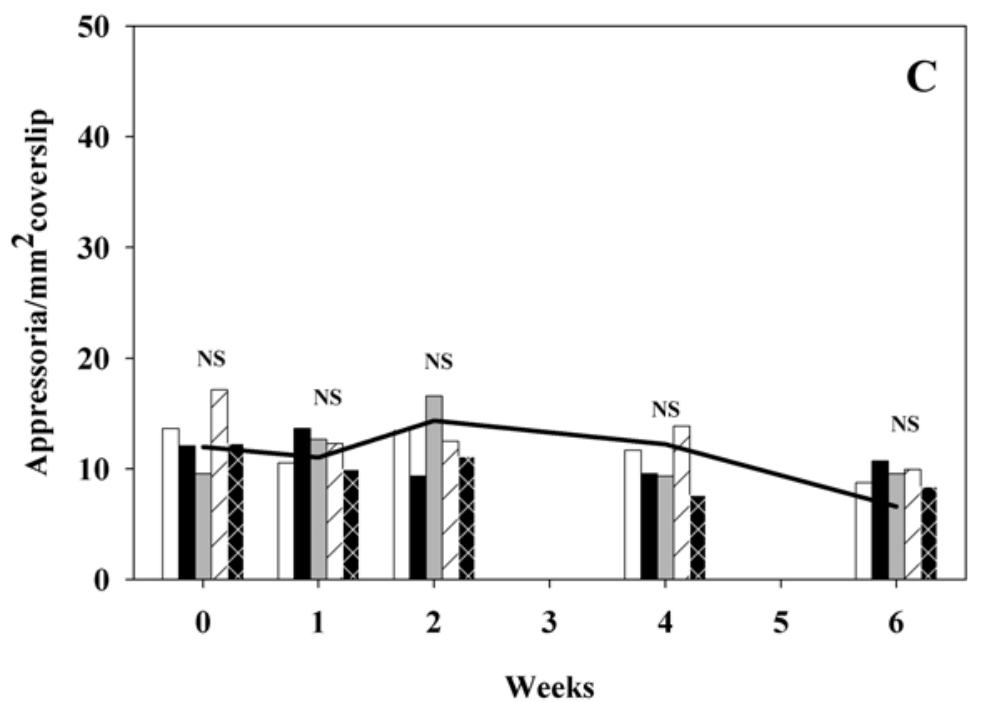

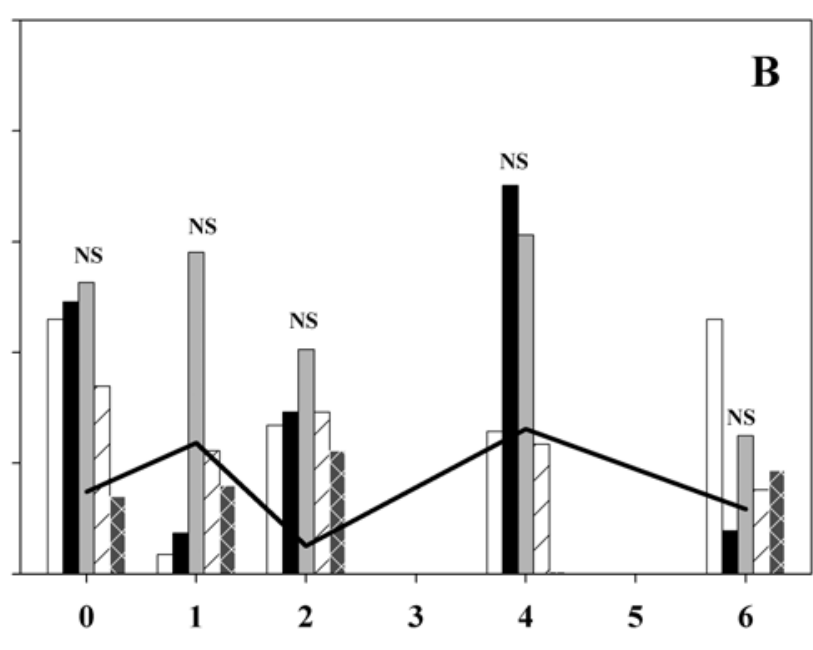

Weeks

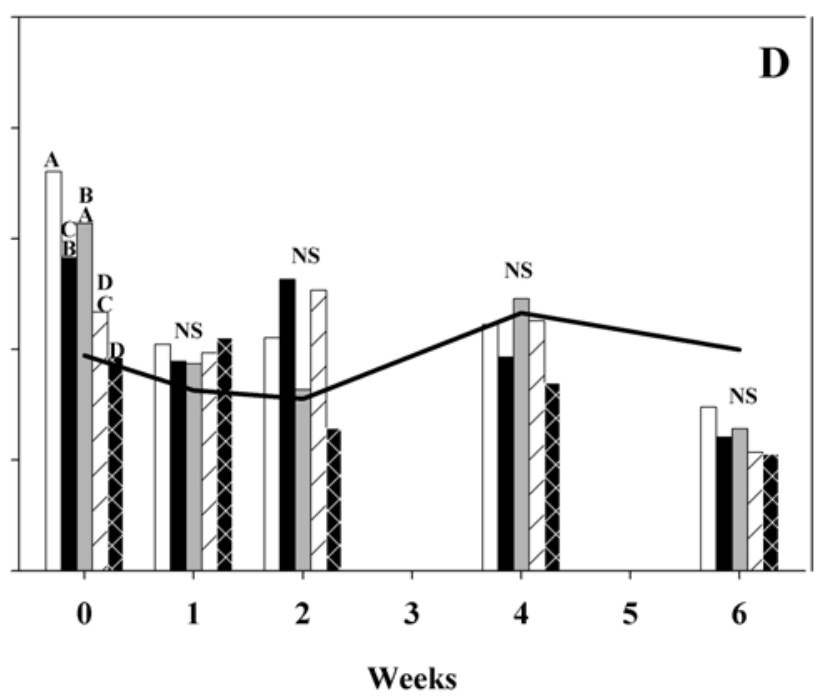

Fig. 3. Colletotrichum acutatum appressoria on leaves in $\mathbf{A}$, run 1 and $\mathbf{B}$, run 2 of the experiment, and on coverslips in $\mathbf{C}$, run 1 and $\mathbf{D}$, run 2 . Leaves and coverslips were sprayed with conidial suspension, incubated for $72 \mathrm{~h}$ at $25^{\circ} \mathrm{C}$ under continuous wetness, and sprayed with water, flower extracts (F1:27 and $\mathrm{F} 1: 81$ ), or leaf extracts (L1:27 and L1:81) after 0 to 6 weeks of exposure to dryness at $25^{\circ} \mathrm{C}$. After treatment application, leaves and coverslips were incubated for $48 \mathrm{~h}$ under continuous wetness at $25^{\circ} \mathrm{C}$. Flower and leaf extracts dilutions are represented by the ratio (wt/vol) of plant material to water. The continuous line represents appressorial populations prior to treatment application and incubation. Bars containing the same letter at each time are not significantly different at $P=0.05$ according to Fisher's least significant difference (LSD) test $(n=2)$. NS $=$ no significant differences within the group. Data presented are means for each experiment. LSD values to compared treatment means within sampling times $0,1,2,4$, and 6 weeks are, respectively A, 17.86, 25.53, 15.38, 10.03, and 17.78; B, 60.23, 25.86, 33.81, 35.24, and 20.85; C, 9.48, 8.88, 4.50, 10.25, and 5.37; and D, 7.50, 14.84, 12.99, 13.47, and 12.74. 
material also has been demonstrated on clothing of fern field workers, where conidia survived for more than 4 weeks (19).

Although stimulation of secondary conidiation by flower extracts was clearly demonstrated, effects of leaf extracts were not as clear. Leaf extracts stimulated secondary conidiation when conidia were germinated in the extracts and when applied to $C$. acutatum populations established on coverslips, but only once when applied to populations already established on leaves. Furthermore, the increase in conidia caused by the leaf extracts was not as pronounced as with flowers. These results may be explained by differences in nutritional composition, and in the balance of inhibitory and stimulatory substances in the leaf and flower extracts (10,25). Germination of $C$. graminicola, for example, was inhibited by substances present in sorghum leaf extracts (25). In our study, inhibitory effects of substances in the leaf extracts may have been counterbalanced by stimulatory substances, resulting in stimulation of secondary conidiation in some cases.

Growth and infection efficacy of several fungi have been shown to depend on the nutritional environment in which inoculum is produced $(2,24,31,32)$. Therefore, further work should compare the viability of conidia produced in different plant extracts and water, and investigate the extract components that are stimulatory to $C$. acutatum. In this study, we focused on the effects of strawberry flower and leaf extracts to represent conditions that may occur before fruit development during the symptomless phase of strawberry anthracnose. Fruit is the preferred plant organ for $C$. acutatum infection; therefore, potential stimulatory effects of fruit extracts on epiphytic behavior of this fungus also should be determined.

The findings of this study enhance understanding of the epiphytic phase of $C$. acutatum on strawberry. Our work not only corroborates earlier studies indicating the capacity of this fungus to persist as an epiphyte $(7,13,14)$, but also suggests that inoculum production on foliage may be greatly increased in the presence of flower extracts. Under field conditions, water-soluble extracts may be deposited onto leaves by rain splashing from flowers, or may be released from flower parts that fall onto leaves during and after flowering. Therefore, it is reasonable to assume that $C$. $a c u$ tatum populations surviving on foliage may be stimulated to produce inoculum by flower extracts under natural conditions. We suggest that these new findings could be valuable when developing management strategies against strawberry anthracnose. For example, fungicide applications aimed at reducing inoculum levels on strawberry plants may prevent multiplication of $C$. acutatum during flowering, thus reducing inoculum available for fruit and flower infections and delaying or preventing subsequent disease development.

\section{ACKNOWLEDGMENTS}

This research was partially funded by grants from the North American Strawberry Growers Association and the Leopold Center for Sustainable Agriculture, as well as a Praxis XXI scholarship to the L. F. S. Leandro from the Fundação para a Ciência e Tecnologia, Portugal. (Portion of Ph.D. research by L. F. S. Leandro.) We thank R. Sakuma, Norcal Inc., for generously supplying strawberry plants for our experiments.

\section{LITERATURE CITED}

1. Agostini, J. P., and Timmer, L. W. 1994. Population dynamics and survival of strains of Colletotrichum gloeosporioides on citrus in Florida. Phytopathology 84:420-425.

2. Banttari, E. E., and Wilcoxson, R. D. 1964. Relation of nutrients inoculum and inoculum concentrations to severity of spring black stem of alfalfa. Phytopathology 54:1048-1052.

3. Boosalis, M. G. 1962. Precocious sporulation and longevity of conidia of Helminthosporium sativum in soil. Phytopathology 52:1172-1177.

4. Dhingra, O. D., and Sinclair, J. B. 1985. Basic Plant Pathology Methods. CRC Press, Inc., Boca Raton, FL.
5. Eastburn, D. M., and Gubler, W. D. 1990. Strawberry anthracnose: Detection and survival of Colletotrichum acutatum in soil. Plant Dis. 74:161-163.

6. Eastburn, D. M., and Gubler, W. D. 1992. Effects of soil moisture and temperature on the survival of Colletotrichum acutatum. Plant Dis. 76:841-842.

7. Freeman, S., Horowitz, S., and Sharon, A. 2001. Pathogenic and nonpathogenic lifestyles in Colletotrichum acutatum from strawberry and other plants. Phytopathology 91:986-992.

8. Freeman, S., and Katan, T. 1997. Identification of Colletotrichum species responsible for anthracnose and root necrosis of strawberry in Israel. Phytopathology 87:516-521.

9. Freeman, S., Nizani, Y., Dotan, S., Even, S., and Sando, T. 1997. Control of Colletotrichum acutatum in strawberry under laboratory, greenhouse and field conditions. Plant Dis. 81:749-752.

10. Grover, R. K. 1971. Participation of host exudate chemicals in appressorium formation of Colletotrichum piperatum. Pages 509-518 in: Ecology of Leaf Surface Microorganisms. T. F. Preece and C. H. Dickinson, eds. Academic Press, London.

11. Howard, C. M., Chandler, C. K., and Albregts, E. E. 1991. Strawberry anthracnose in Florida. Pages 219-220 in: The Strawberry into the 21 st Century: Proc. North Am. Strawberry Conf. 3rd. A. Dale and J. J. Luby, eds. Timber Press, Portland, OR.

12. Howard, C. M., Maas, J. L., Chandler, C. K., and Albregts, E. E. 1992. Anthracnose of strawberry caused by the Colletotrichum complex in Florida. Plant Dis. 76:976-981.

13. Leandro, L. F. S., Gleason, M. L., Nutter, F. W., Jr., Wegulo, S. N., and Dixon, P. M. 2001. Germination and sporulation of Colletotrichum acutatum on symptomless strawberry leaves. Phytopathology 91:659-664.

14. Leandro, L. F. S., Gleason, M. L., Nutter, F. W., Jr., Wegulo, S. N., and Dixon, P. M. 2003. Influence of temperature and wetness duration on conidia and appressoria of Colletotrichum acutatum on symptomless strawberry leaves. Phytopathology 93:513-520.

15. Leandro, L. F. S., Gleason, M. L., Wegulo, S. N., and Nutter, F. W., Jr. 2002. Effects of strawberry plant extracts on conidiation and appressorial production by Colletotrichum acutatum. (Abstr.) Phytopathology 92(suppl.):S45.

16. Legard, D. E., Whidden, A. J., and Chandler, C. K. 1997. Incidence and occurrence of strawberry diseases in Florida from 1991-1996. Adv. Strawberry Res. 16:35-47.

17. McInnes, T. B., Black, L. L., and Gatti, J. M., Jr. 1992. Disease-free plants for management of strawberry anthracnose crown rot. Plant Dis. 76:260-264.

18. Muirhead, I. F. 1998. The role of appressorial dormancy in latent infection. Pages 155-167 in: Microbial Ecology of the Phylloplane. J. P. Blakeman, ed. Academic Press, New York.

19. Norman, D. J., and Strandberg, O. J. 1997. Survival of Colletotrichum acutatum in soil and plant debris of leatherleaf fern. Plant Dis. 81:11771180.

20. Pantidou, M. E., and Schroeder, W. T. 1955. Foliage as a source of secondary inoculum for tomato anthracnose. Phytopathology 45:338-345.

21. Parbery, D. G. 1981. Biology of anthracnose on leaf surfaces. Pages 135154 in: Microbial Ecology of the Phylloplane. J. P. Blakeman, ed. Academic Press, New York.

22. Parbery, D. G., and Blakeman, J. P. 1978. Effect of substances associated with leaf surfaces on appressorium formation by Colletotrichum acutatum. Trans. Br. Mycol. Soc. 70:7-19.

23. Peterson, R. A. 1977. Green leaves as a source of inoculum for anthracnose fungi, with special reference to Colletotrichum acutatum on strawberry. M.S. thesis. Department of Botany, University of Queensland, Australia.

24. Schisler, D. A., Jackson, M. A., and Bothast, R. J. 1991. Influence of nutrition during conidiation of Colletotrichum truncatum on conidial germination and efficacy in inciting disease in Sesbania exaltata. Phytopathology 81:458-461.

25. Sharma, J. K., and Sinha, S. 1971. Effects of leaf exudates of sorghum varieties varying in susceptibility and maturity on the germination of conidia of Colletotrichum graminicola (Ces.) Wilson. Pages 597-601 in: Ecology of Leaf Surface Microorganisms. T. F. Preece and C. H. Dickinson, eds. Academic Press, London.

26. Slade, S. J., Harris, R. F., Smith, C. S., and Andrews, J. H. 1987. Microcyclic conidiation and spore-carrying capacity of Colletotrichum gloeosporoides on solid media. Appl. Environ. Microbiol. 53:2106-2110.

27. Smith, B. J. 1998. Anthracnose fruit rot (black spot). Pages 31-33 in: Compendium of Strawberry Diseases. 2nd ed. J. L. Maas, ed. The American Phytopathological Society, St. Paul, MN.

28. Smith, B. J., and Black, L. L. 1990. Morphological, cultural and pathogenic variation among Colletotrichum species isolated from strawberry. Plant Dis. 74:69-76. 
29. Smith, J. E., Anderson, J. G., Deans, S. G., and Berry, D. R. 1981. Biochemistry of microcycle conidiation. Pages 329-356 in: Biology of Conidial Fungi. G. T. Cole and B. Kendrick, eds. Academic Press, New York.

30. Timmer, L. W., and Brown, G. E. 2000. Biology and control of anthracnose diseases of citrus. Pages 300-316 in: Colletotrichum: Host Specificity, Pathology, and Host-Pathogen Interaction. D. Prusky, S. Freeman, and M. B. Dickman, eds. The American Phytopathological Society, St. Paul, MN.

31. Trainor, M. J., and Martinson, C. A. 1978. Nutrition during spore production and the inoculum potential of Helminthosporium maydis race $\mathrm{T}$. Phytopathology 68:1049-1053.
32. Weinhold, A. R., and Bowman, T. D. R. L. 1969. Virulence of Rhizoctonia solani as affected by nutrition of the pathogen. Phytopathology 59:1601-1605.

33. Wilson, L. L., Madden, L. V., and Ellis, M. A. 1992. Overwinter survival of Colletotrichum acutatum in infected strawberry fruit in Ohio. Plant Dis. 76:948-950.

34. Wise, K. A., Leandro, L. F. S., Wegulo, S. N., and Gleason, M. L. 2002. Survival and dispersal of Colletotrichum acutatum on strawberry leaves under field conditions. (Abstr.) Phytopathology 92(suppl.):S87.

35. Zulfiqar, M., Brlansky, R. H., and Timmer, L. W. 1996. Infection of flower and vegetative tissues of citrus by Colletotrichum acutatum and C. gloeosporioides. Mycologia 88:121-128. 